Research Article

\title{
Constant Frequency Control Strategy of Microgrids by Coordinating Energy Router and Energy Storage System
}

\author{
Zheng Lan, ${ }^{1}$ Junzhang Wang $\mathbb{D},{ }^{1}$ Jinhui Zeng $\mathbb{D},{ }^{1}$ Dong He, ${ }^{1}$ Fan Xiao, ${ }^{2}$ and Fei Jiang ${ }^{3}$ \\ ${ }^{1}$ College of Electrical and Information Engineering, Hunan University of Technology, Zhuzhou, Hunan, China \\ ${ }^{2}$ College of Electrical and Information Engineering, Hunan University, Changsha, Hunan, China \\ ${ }^{3}$ College of Electrical and Information Engineering, Changsha University of Science \&Technology, Changsha, Hunan, China
}

Correspondence should be addressed to Jinhui Zeng; zjh@hut.edu.cn

Received 19 May 2020; Accepted 15 July 2020; Published 14 August 2020

Guest Editor: Hou-Sheng Su

Copyright (C) 2020 Zheng Lan et al. This is an open access article distributed under the Creative Commons Attribution License, which permits unrestricted use, distribution, and reproduction in any medium, provided the original work is properly cited.

In this paper, a constant frequency control strategy of a microgrid by coordinating energy router (ER) and energy storage system is proposed to solve the frequency fluctuation problem of microgrid, in which ER is the core of the energy management system. The interface converter of ER connected to the microgrid adopts the virtual synchronous generator (VSG) control, which adjusts the mechanical reference power in real time according to the state of charge (SOC) of the energy storage. The energy storage system adopts constant voltage and constant frequency (V/F) control to maintain the frequency and voltage stability of microgrid. The energy storage system with fast charge and discharge responds to the power fluctuation of the microgrid. ER maintains the stability of the energy storage capacity through bidirectional power regulation. When the energy storage system fails, the frequency and voltage droop characteristics of ER controlled by VSG will play a role in maintaining the stable operation of the microgrid. By the coordinating control strategy, the maximum efficiency of intermittent distributed energy can be guaranteed, and the stability, reliability, and grid-connection friendliness of the microgrid operation can be improved. Simulation results prove the effectiveness of the proposed control strategy.

\section{Introduction}

In recent years, due to the rapid development of distributed power generation technology, a large number of distributed new energy grid-connection requirements have emerged. However, due to the intermittent and fluctuating characteristics of new energy sources such as photovoltaics, a large number of new energy sources directly incorporated into the grid will bring severe challenges to the stability of traditional power systems. The microgrid has become one of the effective ways to dissipate distributed energy sources (DER) $[1,2]$. Among them, microgrids with ER as the core have been proposed successively [3-5].

In this kind of microgrid, DER, local loads, and battery energy storage system (BESS) are usually connected to the main grid through the low-voltage AC interface provided by the ER. The ER transmits and manages the surplus and deficit power of distributed power after local absorption and completes the functions of initiative adjustment of electric energy and adjustment of electric power quality.

To control the frequency stability of the microgrid, we need an effective control strategy. The load in the microgrid is uncontrollable. At the same time, to avoid wind and light abandonment, distributed power generation units often use maximum power point tracking (MPPT) control $[6,7]$. Therefore, neither load nodes nor distributed generation nodes can participate in frequency regulation without the use of deloading control. To improve the operational performance of the microgrid, we used the energy storage system as an energy buffer device to suppress power fluctuations. At the same time, the energy storage system is jointly powered with ER and DER [8-10]. Therefore, the microgrid with the ER as the core needs to control the energy storage unit node and the ER node to achieve the frequency stability of the microgrid. How to coordinate and control ER, distributed energy, and 
related energy storage devices has become an urgent problem to be solved.

In the current research, there are two main research approaches to ensure the stable operation of the microgrid in the complex network connected with many distributed power sources. One is to make the output voltage and frequency of each distributed network node be the same. Many scholars have proposed the edge consensus algorithms $[11,12]$, which ensure that the network system can still maintain the consistency of output voltage and frequency of each node under various disturbances; to improve the stability of the system, many scholars put forward the global robust control algorithm of the network system [13]. The other is by coordinating the power of the units in the microgrid. The authors in $[14,15]$ propose to combine the energy storage system with wind turbines and other DER to reduce the power fluctuation of the microgrid. This scheme can make the wind turbine and other DER output at maximum power, and the energy storage system quickly absorbs or releases the surplus and deficiency power of the microgrid, which can avoid the frequency instability of microgrid caused by the power fluctuation of DER. The authors in [16] propose a master-slave control strategy, which controls the output of the ER as a constant voltage and constant frequency voltage source and the BESS control as a current source with controllable power. Under this control strategy, the microgrid can keep the frequency constant. However, because there is no local frequency variable as the judgment index of ER and BESS outputs, power allocation can only be implemented through the upper energy management system, the control is complex and transitionally dependent on communication, and the reliability is not high. Also, to solve the problem that the network system can still achieve synchronization in the case of coupling resonance or disturbance, the authors in $[17,18]$ propose adaptive synchronization algorithms, respectively. Lv et al. [19] introduced the VSG control technology into the control of the ER interface, which enhanced the inertial damping of the system and reduced the impact of the wind-wave output on the main grid. However, the coordinated operation of the ER and energy storage was not involved in the paper. However, the coordinated operation of the ER and the energy storage system was not involved in this paper.

Based on the above analysis, this paper proposes a constant frequency control strategy of the microgrid by coordinating the ER and the energy storage system. We can refer to the method of virtual reactance to limit fault current in [20] and introduce VSG control into the low-voltage AC output interface of ER to increase the inertia of the microgrid system and reduce the large frequency fluctuations caused by the load disturbance. The ER adjusts the SOC of the energy storage based on weak communication. The BESS adopts constant voltage and frequency $(\mathrm{V} / \mathrm{F})$ control to provide constant voltage and frequency for the microgrid. On the one hand, the proposed control strategy can solve the problem of frequency fluctuation of microgrid and, at the same time, avoid overcharging or overdischarging of the energy storage system. On the other hand, when ER or RSS fails, there is no need to switch control strategies, ensuring the smooth operation of the microgrid. Finally, the operation results of the proposed control strategy are verified by simulation.

\section{Description of the Microgrid System}

The typical microgrid structure with the ER as the core is shown in Figure 1(a).

DC loads such as charging pile need to be extracted through AC/DC converter, and AC load such as the AC motor can be directly connected with a bus. The DER such as wind power and photovoltaic power is connected to an $\mathrm{AC}$ bus through $\mathrm{DC} / \mathrm{AC}$ and $\mathrm{AC} / \mathrm{DC} / \mathrm{AC}$ converters, respectively, and the BESS is connected to microgrid through DC/ AC converters to jointly supply power for the local loads. As an energy hub, ER connects the main grid and the microgrid, and its structure is shown in Figure 1(b). ER consists of three parts: the input stage, isolation stage, and output stage. The input stage is cascade-connected by the $\mathrm{H}$-bridge $\mathrm{AC} / \mathrm{DC}$ rectifier, which can realize different levels of voltage access of the main grid. The isolation stage is composed of a bidirectional active full-bridge DC/DC converter in parallel, which plays the role of bidirectional power transmission, changing voltage, and isolating the main grid and microgrid. The output stage is a three-phase bridge inverter structure that provides a low-voltage $\mathrm{AC}$ interface to connect to the AC bus of the microgrid.

2.1. Principle of System Coordination Operation. In the overall control of ER, the input stage mainly controls the voltage stability of the capacitance $C_{H N}$ at the high voltage side, which plays a rectifying role; the isolation stage controls the voltage stability of the capacitance $C_{L}$ at the low-voltage side, which plays a role of voltage level exchange, bidirectional energy flow, and isolation between the input stage and the output stage [21]; the output stage inverts the direct current into three-phase AC output. As the control strategy of this paper is mainly realized by the ER output stage, the following research on the analysis of the coordinated operation principle is mainly carried out in the output stage. In the whole microgrid topology, the MPPT control is used to ensure the maximum power output of distributed generation. When both ER and BESS are working properly, the BESS is used as the balance node to provide voltage and frequency support and respond to the unbalanced power of DER and load quickly. ER, as the PQ node, adjusts the power output according to the SOC of BESS in real time to ensure that the SOC of the BESS is always in the normal range. We can take Figure 2 as an example to analyze the coordination process of ER and BESS.

Suppose there is a mismatch between DER output and load demand at some time, such as a sudden increase in load or a sudden decrease in DER output, and a power deficit $P_{e q}$ will occur in the microgrid:

$$
P_{e q}=\sum_{i \in S_{L}} P_{L i}-\sum_{i \in S_{R S}} P_{R S i}
$$




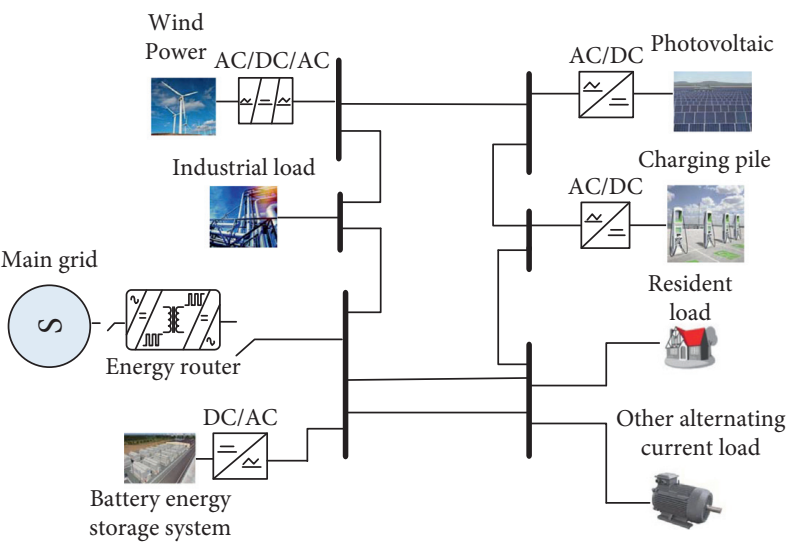

(a)

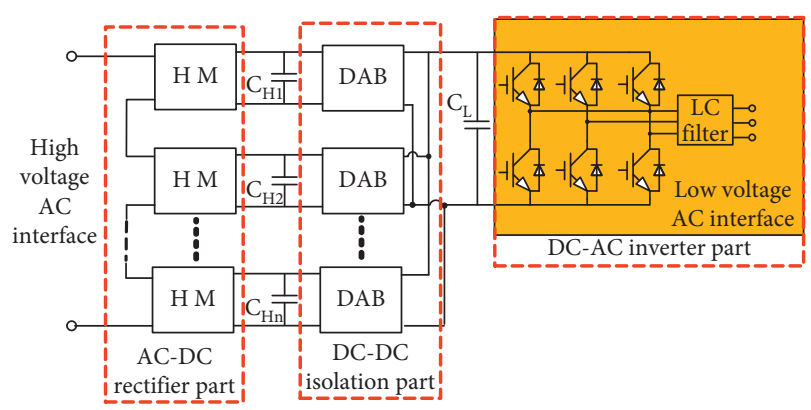

(b)

Figure 1: System structure of the microgrid and topology of the energy router: (a) microgrid system structure; (b) topology of energy router.

where $P_{e q}$ is the net load of microgrid, $P_{L i}$ is each load in the system, $P_{R S i}$ is the power output of various renewable energy sources, $S_{L}$ is the system load collection, and $S_{R S}$ is the collection of renewable energy sources within the system.

If sufficient power is not replenished quickly, the frequency of the microgrid will fluctuate. When the power vacancy is too large, the frequency will drop sharply and even affect the stability of the microgrid. At this time, BESS rapidly releases the electric energy to ensure the active power balance. As the output power of BESS causes its SOC to be lower than the reference value, to maintain the stability of the energy storage capacity, ER reflects virtual inertia and slowly increases its active power output to share the power gap and charges the energy storage system. Through the adjustment of the ER, the electric energy released by BESS gradually reduces to zero and then absorbs the power to make its capacity recover. When the SOC returns to the reference value, the BESS output is zero, and the power gap is finally borne by the ER. Similarly, when DER output increases or load decreases, resulting in the power surplus of the microgrid, BESS quickly absorbs the surplus power, while ER slowly adjusts the SOC of the energy storage and finally transmits the surplus power to the main grid.

When the BESS fails and stops working, ER is naturally switched to a balance node and simulates the droop characteristics of synchronous generators to support the basic operation of the microgrid.

\subsection{Coordination Control Strategy between the ER and the BESS}

2.2.1. Control of the BESS. The BESS is composed of a battery, interface inverter, and LC filter. The output interface is connected with the $\mathrm{AC}$ bus of the microgrid. The purpose of BESS control is to provide stable voltage and frequency support for the microgrid and rapidly output according to the fluctuating power of DER or load. Its interface converter adopts constant voltage and constant frequency (V/F) control based on voltage and current double closed-loop [22]. The control block diagram is shown in Figure 3.

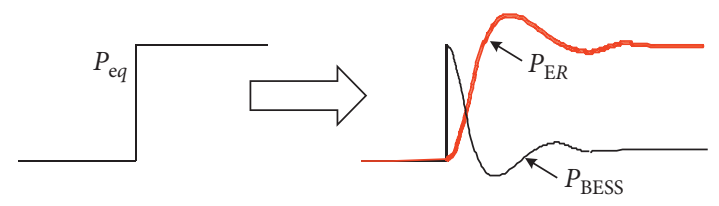

Figure 2: Schematic diagram of the coordinated control.

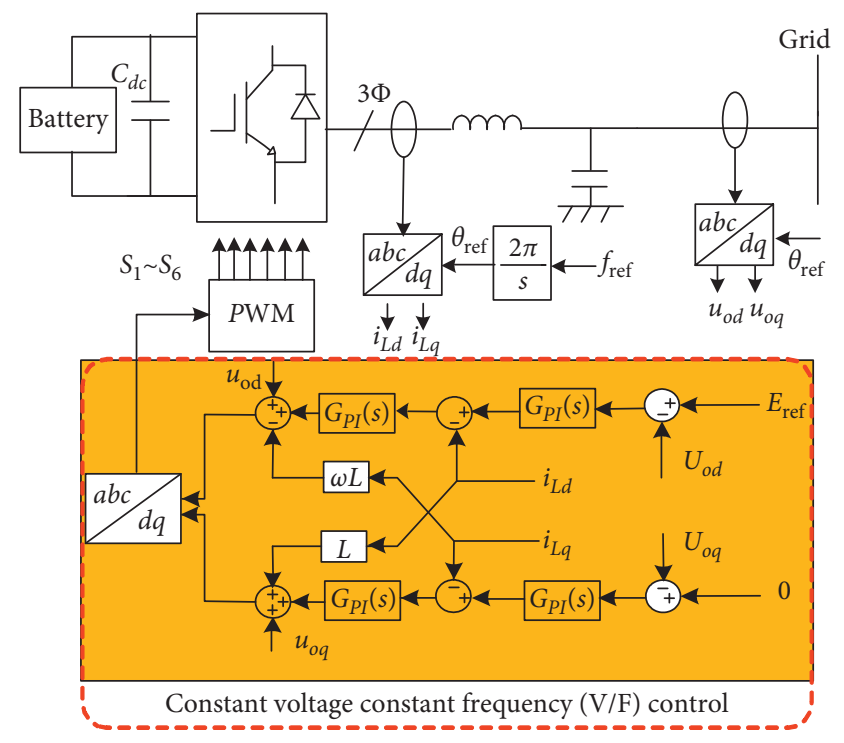

Figure 3: Control scheme of the BESS.

In Figure $3, i_{L a b c}$ is the filtering inductance current, $u_{o a b c}$ is the output voltage of the interface converter, and $E_{\text {ref }}$ and $f_{\text {ref }}$ are the given voltage amplitude and frequency reference values, respectively. The phase angle $\theta_{\text {ref }}$ needed for the coordinate transformation is obtained by integrating the reference frequency. After the $a b c / \mathrm{d} q$ coordinate transformation of the output voltage, the $u_{o d}$ and $u_{o q}$ components are obtained, which are compared with the voltage reference $E_{\text {ref }}$ and 0, respectively, and the error is controlled by PI, so as to obtain the reference signals of the inner ring, $i_{d \text { ref }}$, and $i_{q \text { ref }}$. Voltage modulating signal $u_{\text {ref }}$ was obtained through PI control, current loop, and dq/abc transformation of inner 
loop current reference signal $\left(i_{L d}\right)$ and filter inductance current component $\left(i_{L q}\right)$.

2.2.2. Control of the ER. The objective of the input stage control of the ER is to stabilize the voltage of the capacitor on the high voltage side. To improve the control accuracy, the double closed-loop control is adopted. The voltage of the measured capacitor is compared with the voltage of the given capacitor to form a voltage outer loop to stabilize the voltage of the capacitor on the high side. The current inner loop can control the AC side current waveform, realize the control of the power factor of the input stage, and at the same time, can improve the control accuracy and response speed. The isolation stage adopts phase shift control [23] to realize the two-way flow of power.

The output stage of the ER adopts VSG control $[19,24,25]$. The VSG introduces the rotor motion equation and electromagnetic transient equation of the synchronous machine into the control of the output stage. It can make the inverter output of the output stage of ER equivalent to a voltage source with controllable power angle and voltage amplitude and make its output characteristics to simulate synchronous generator so that the inverter of the output stage of ER also has inertia, which can adjust voltage and frequency automatically. Since there are many studies on VSG at present, only the control block diagram (Figure 4) is given here, and the specific principle will not be detailed.

\subsection{Coordination Control between the ER and the BESS.} The BESS provides constant voltage and frequency for the system in the whole microgrid, and its fast charging and discharging capacity can also quickly respond to the power fluctuations of DER. However, BESS can neither maintain its SOC nor provide frequency droop characteristics for the system. To overcome this problem, the coordinated operation of the ER and BESS is realized by designing the mechanical reference power in VSG control. The specific control block diagram is shown in Figure 5.

When BESS is in normal operation, the switch is connected to point a. Since BESS provides a stable frequency for the microgrid at this time, the VSG power-frequency $(P-f)$ regulation characteristic does not work. ER outputs power according to the mechanical reference power $P_{\text {ref }}$ and adjusts the energy storage capacity, and the $P_{\text {ref }}$ expression is

$$
P_{\text {ref }}=P_{o}+\Delta P
$$

where $P_{o}$ is the base point of the running power command, which is estimated by the difference between the predicted value of the DER average output and the load and $\triangle P$ is the adjusted power that the ER needs to output to maintain the stability of the energy storage capacity. Since the SOC can represent the remaining capacity of the battery, if the instantaneous value of the state of charge of the battery can track its reference value $\mathrm{SOC}_{\text {ref }}$, the stability of the BESS capacity can be achieved. Therefore, closed-loop control is performed on the SOC. The acquired SOC instantaneous value is transmitted to the controller of the ER to form

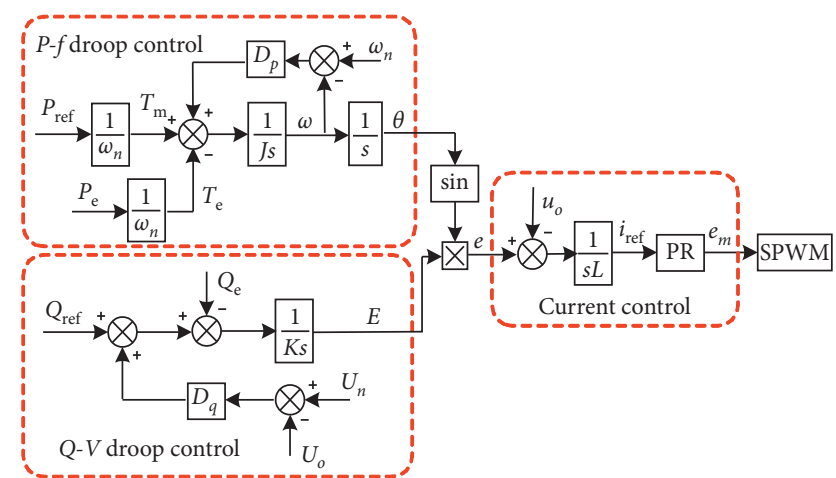

FIGURE 4: Control scheme of the VSG for the output stage of the ER.

closed-loop feedback with the $\mathrm{SOC}_{\text {ref }}$. After the PI controller controls the feedback output, the adjusted power $\Delta P$ can be obtained:

$$
\Delta P=\left(K_{p}+\frac{K_{i}}{s}\right)\left(\mathrm{SOC}_{\mathrm{ref}}-\mathrm{SOC}\right),
$$

where $K_{p}$ and $K_{i}$ are the proportional coefficients and integral coefficient of PI controller, respectively.

In order to prevent the transmission power of the ER from exceeding the rated range in extreme cases, $\mathrm{SOC}_{\text {ref }}$ in this paper needs to be a reference value that can be dynamically adjusted. By changing $\mathrm{SOC}_{\text {ref }}$, ER can control the active power output of BESS. For example, when faced with extreme weather without wind and light, the DER stops generating power, resulting in an imbalance between microgrid supply and demand. To prevent ER transmission power from exceeding the rated range, we can reduce the $\mathrm{SOC}_{\text {ref }}$ so that BESS can release part of the electric energy and share the transmission power of the ER.

As you can see from Figure 5, you can adjust the $P_{\mathrm{BESS}}$, ref parameter to achieve the adjustment of $\mathrm{SOC}_{\text {ref }}$. $\mathrm{SOC}_{i}$ is the initial value of the state of charge, which is determined by the initial state of BESS, so $\mathrm{SOC}_{i}$ cannot be adjusted. Adjusting the parameter of $P_{\mathrm{BESS} \text {, ref }}$ is essentially adjusting the slope of $\mathrm{SOC}_{\text {ref }}$. This can be explained by the following equation. According to the relationship between the active power output of the battery and its state of charge $[26,27]$,

$$
\begin{aligned}
\mathrm{SOC} & =\mathrm{SOC}_{i}-\int \frac{I_{d c}}{\mathrm{Q}} \mathrm{d} t, \\
I_{d c} & =\frac{P_{\mathrm{BESS}}}{V_{d c}}, \\
Q & =\frac{I_{d c, \text { rate }} C_{\mathrm{BESS}, \text { rate }}}{P_{\text {BESS,rate }}},
\end{aligned}
$$

where $\mathrm{SOC}_{i}$ is the initial value of the state of charge, $I_{d c}$ is the output current of the DC side of the BESS, $V_{d c}$ is the DC side voltage of the BESS, $P_{\mathrm{BESS}}$ is the active power output of the BESS, $I_{d c \text {,rate }}$ is the rated value of the output DC, $C_{\mathrm{BESS}, \text { rate }}$ is the rated capacity of the BESS, and $P_{\mathrm{BESS}, \text { rate }}$ is the rated active power of the BESS. Substituting equations (5) and (6) into equation (4), we can get 


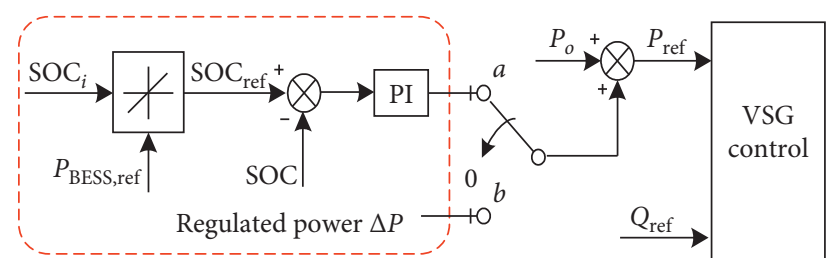

FIgure 5: Power control scheme between ER and BESS.

$$
\mathrm{SOC}=\mathrm{SOC}_{i}-\int \frac{P_{\mathrm{BESS}, \text { rate }} P_{\mathrm{BESS}}}{V_{d c} I_{d c, \text { rate }} C_{\mathrm{BESS}, \text { rate }}} \mathrm{d} t .
$$

Differentiating both sides of equation (7), we get

$$
\frac{\mathrm{d} \text { SOC }}{\mathrm{d} t}=-K_{c} P_{\mathrm{BESS}}
$$

where $K_{c}=P_{\text {BESS,rate }} / V_{d c} I_{d c \text {,rate }} \cdot C_{\text {BESS }}$, rate because the $V_{d c}$ is approximately constant, and $K_{c}$ can be equivalent to constant. It is not difficult to find from equation (8) that BESS's active power output value $P_{\text {BESS }}$ can be controlled by changing the slope $\mathrm{dSOC} / \mathrm{d} t$. Corresponding to the control in Figure $5, P_{\mathrm{BESS} \text {, ref }}$ is the active power that ER wants BESS to share. Change the slope of $\mathrm{SOC}_{\text {ref }}$ by setting $P_{\mathrm{BESS}}$, and then, change the value of $\mathrm{SOC}_{\text {ref }}$ dynamically. In this way, the active output of the ER can be adjusted to control the BESS to share the desired active power $P_{\text {BESS, ref }}$.

Since the frequency of the microgrid is only supported by the BESS, the stability of the microgrid will be greatly threatened when the BESS fails. To solve this problem, the switch design is carried out in the coordinated control strategy of ER and BESS. When BESS faults, the ER will receive the fault information transmitted from BESS through weak communication, and the switch will be connected to the point $b$. The BESS no longer provides frequency and voltage support for the microgrid. ER based on VSG control will adjust the frequency and voltage of the microgrid according to the active power-frequency droop characteristic and the reactive power-voltage droop characteristic and provide certain inertia for the system to enhance the frequency stability of the system. From the active frequency control loop in Figure 4, the frequency droop coefficient $D_{P}$ can be expressed by

$$
D_{P}=\frac{\Delta T}{\Delta \omega}=\frac{\Delta P}{\omega_{n} \Delta \omega}=\frac{\Delta P}{4 \pi^{2} f_{n} \Delta f} .
$$

In the above formula, $\Delta f$ is the variation range of the frequency offset of the microgrid, $f_{n}$ is the rated value of the microgrid frequency $(50 \mathrm{~Hz})$, and $\Delta P$ is the active power variation of the corresponding output of the ER.

\section{Simulation Results}

To verify the correctness and effectiveness of the proposed control strategy, we built a microgrid simulation model under the MATLAB/Simulink platform and set different working conditions for simulation verification. The DER in the model includes wind turbines and photovoltaics, and the energy storage system uses currently widely used lead-acid batteries. When the rated frequency of the microgrid is $50 \mathrm{~Hz}$, the rated capacity of ER is $100 \mathrm{~kW}$, and the frequency variation range is $\pm 0.5 \mathrm{~Hz}$, From equation (9), we can calculate that the frequency droop coefficient is $50.67 \mathrm{Nm} \cdot \mathrm{s} /$ rad. The other main parameters of the simulation are shown in Table 1.

3.1. Working Condition 1: Random Fluctuation of Wind and Solar. As shown in Figure 6(a), the wind power fluctuation ranges from $47 \mathrm{~kW}$ to $83 \mathrm{~kW}$, and the photovoltaic power fluctuation ranges from $18 \mathrm{~kW}$ to $33 \mathrm{~kW}$. The total load demand is $100 \mathrm{~kW}$. The output active power and SOC waveform of BESS and ER are shown in Figures 6(b)$6(d)$, respectively. It can be seen that when the output power of wind energy and photovoltaic light fluctuates, BESS can quickly respond to the unbalanced power of wind and light to ensure the balance of supply and demand in the microgrid, while ER reflects the virtual inertia to slowly adjust the output power according to SOC, support BESS operation, and maintain its SOC stability. Due to fluctuations in the output power of wind and photovoltaic, the SOC of BESS fluctuates. As can be seen from Figure 6(c), the frequency of the microgrid is stable at $50 \mathrm{~Hz}$ and hardly affected by the fluctuation of the landscape.

3.2. Working Condition 2: Sudden Change in the Load. To avoid disturbance of operation effect, the wind and sunlight output is constant with average output. At $3 \mathrm{~s}$, the load of microgrid decreases sharply from $100 \mathrm{~kW}$ to $90 \mathrm{~kW}$. At $3 \mathrm{~s}$, due to sudden load reduction, $10 \mathrm{~kW}$ of surplus power appeared in the microgrid. At this time, BESS quickly absorbed the $10 \mathrm{~kW}$ of surplus power, and ER slowly reduced the power output. Finally, the output power of both BESS and ER tends to zero, as shown in Figure 7(a). At this time, all the loads in the microgrid are provided by wind power and photovoltaic. As can be seen from Figure $7(\mathrm{~b})$, the SOC is gradually higher than the reference value at $3 \mathrm{~s}$ as BESS begins to absorb electricity energy.

The dynamic process of sudden changes in load can be seen more clearly that BESS has a fast output power, which can well suppress the frequency fluctuation caused by a sudden decrease in load, as shown in Figure 7(c). The ER output power waveform is smooth and slow, which effectively alleviates the influence of large power fluctuations on the main grid.

3.3. Working Condition 3: BESS Failure and ER Runs Independently. To simulate the actual situation, wind energy and photovoltaic energy still fluctuate by the law in working condition 1 . When set at $8 \mathrm{~s}$, the operation stopped due to the failure of the BESS. In the coordinated control between ER and BESS, when the ER receives the fault information transmitted from BESS through weak communication, the switch is connected from point $a$ to point $b$ (as shown in 
TABLE 1: Key parameters of the simulation.

\begin{tabular}{lc}
\hline Parameter & Value/unit \\
\hline Maximum fluctuation of the active power of the photovoltaic & $33 \mathrm{~kW}$ \\
Maximum fluctuation of the active power of the wind power & $83 \mathrm{~kW}$ \\
Average active power output of the photovoltaic & $25 \mathrm{~kW}$ \\
Average active power output of the wind power & $65 \mathrm{~kW}$ \\
The capacity of the battery energy storage system & $5 \mathrm{Ah}$ \\
Load & $0-100 \mathrm{~kW}$ \\
Rated power of the ER & $100 \mathrm{~kW}$ \\
$K_{P I}$ of the PI controller & 110 \\
$I_{P I}$ of the PI controller & 210 \\
Rated frequency of the microgrid & $50 \mathrm{~Hz}$ \\
Rated line voltage & $380 \mathrm{~V}$ \\
Droop control coefficient & $50.67 \mathrm{Nm} \cdot \mathrm{s} / \mathrm{rad}$ \\
\hline
\end{tabular}

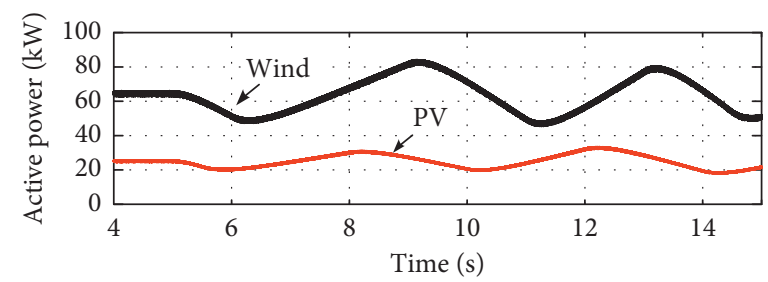

(a)

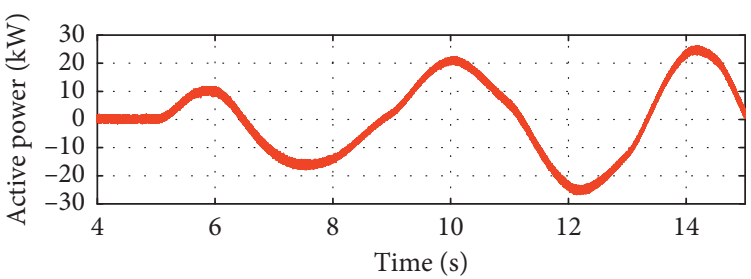

(b)
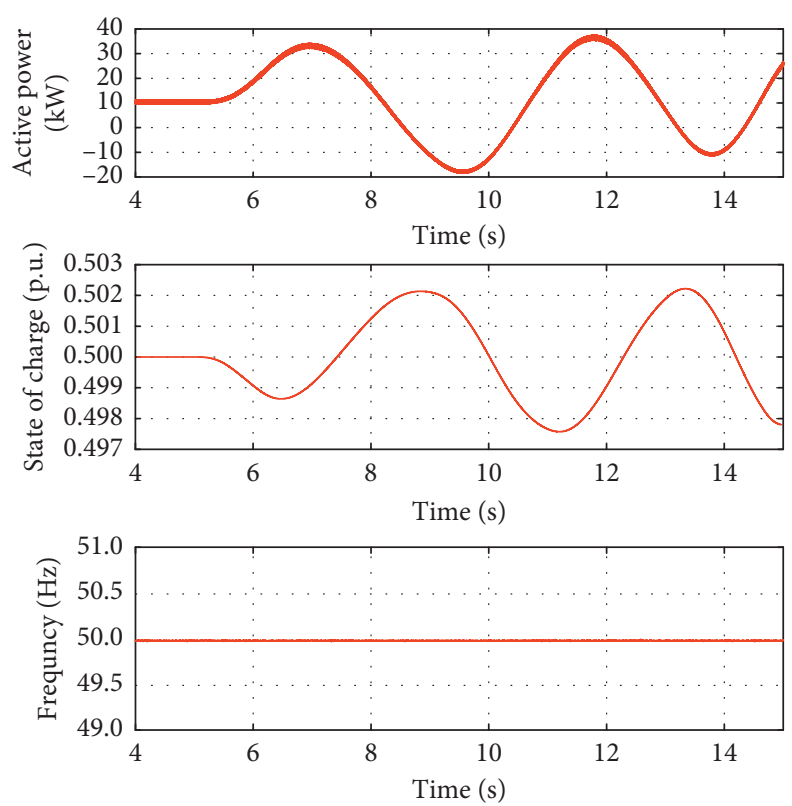

(c)

FIGURE 6: Simulation waveforms for wind and photovoltaic fluctuations: (a) active power of wind and photovoltaic; (b) active power of BESS; (c) frequency of microgrid.

Figure 5), switching to the independent operation mode of ER.

As can be seen from Figures 8(a) and 8(b), initially the BESS responded quickly to the wind and light fluctuations, and the system frequency fluctuations were small. At $8 \mathrm{~s}$, the BESS fails, and its output power is zero. At this time, the ER controlled by VSG responds to the fluctuation of wind and light and adjusts the frequency of the microgrid according to the characteristics of the droop frequency. Figure 8(c) is the waveform of the SOC of BESS. Since the BESS output power is zero at $8 \mathrm{~s}$, the SOC will not change after $8 \mathrm{~s}$. Figure $8(\mathrm{~d})$ shows the voltage waveform of the AC bus of the microgrid. We can see that the voltage waveform is smooth and the amplitude is stable during the switching process, which realizes the smooth operation of the microgrid during the BESS fault. 


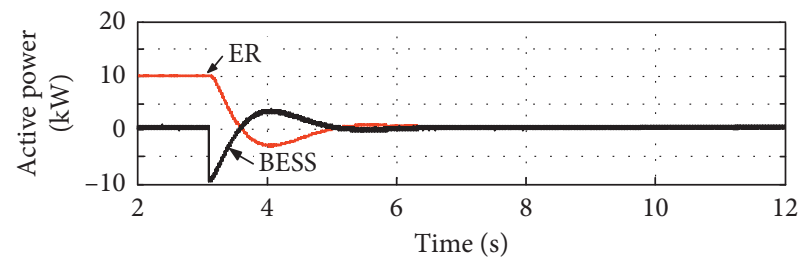

(a)

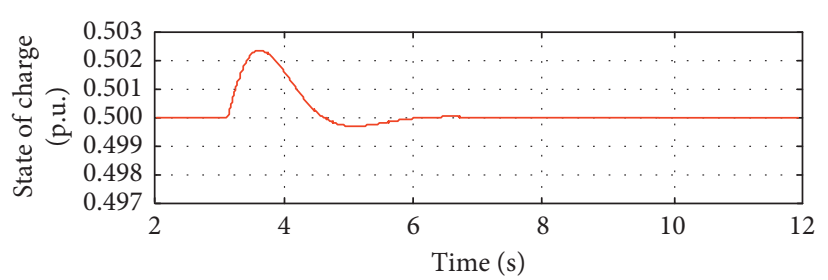

(b)

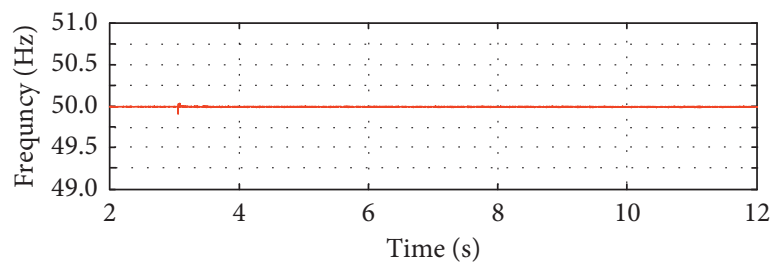

(c)

FIGURE 7: Simulation waveforms under load decrease: (a) active power of ER and BESS; (b) state of charge; (c) frequency of microgrid.

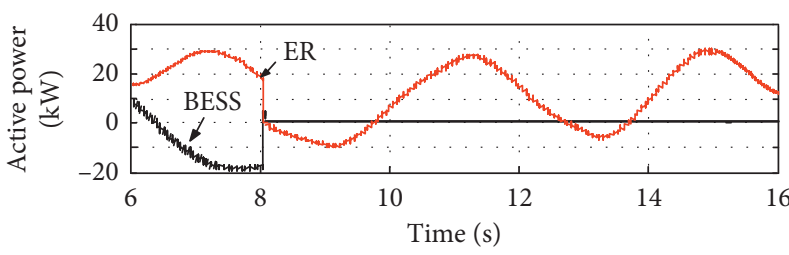

(a)

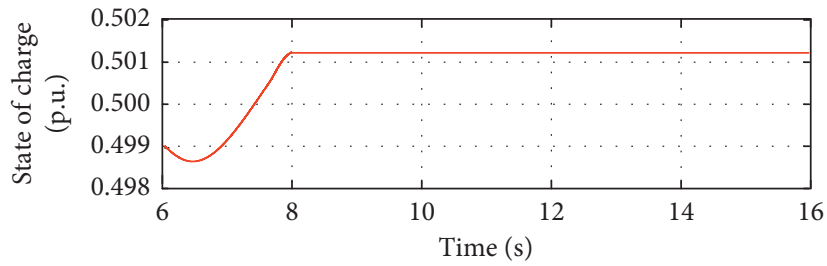

(c)

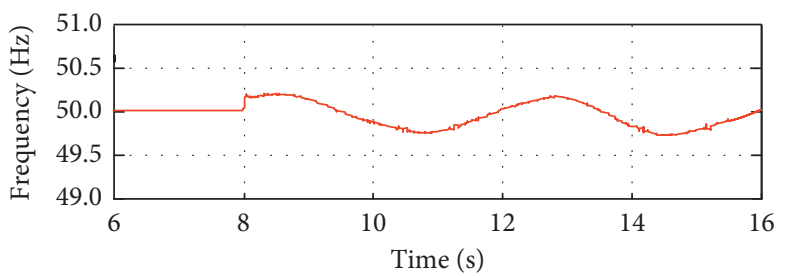

(b)

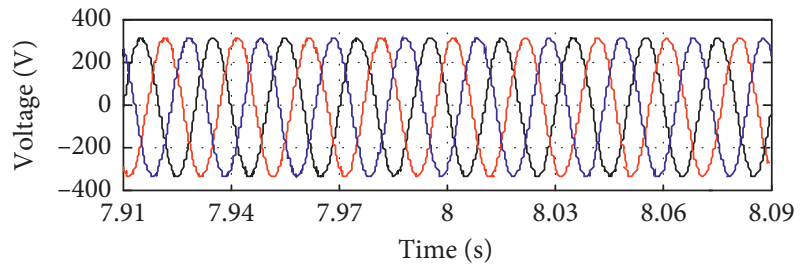

(d)

FIGURE 8: Simulation waveform when BESS fails: (a) active power of ER and BESS; (b) frequency of microgrid; (c) state of charge; (d) voltage of microgrid AC bus.

\section{Conclusion}

To control the frequency of the microgrid with the ER as the core, we propose a constant frequency control strategy coordinated by the ER and BESS. The principle of coordinated operation of ER and BESS under the microgrid system structure has been described and analyzed; meanwhile, the specific coordinated control strategy of ER and BESS has been given. Finally, MATLAB/Simulink was used to build a simulation model and conduct simulation verification. We can get the following conclusions:

(1) BESS uses constant voltage and constant frequency control to stabilize the frequency at the rated value and can quickly respond to power fluctuations to ensure the active balance of the microgrid.

(2) ER maintains the stability of energy storage capacity and controls the output power of BESS by adjusting the reference value of SOC. The virtual synchronous motor control makes the interface of the ER have the virtual inertia of the synchronous motor, which ensures the smooth exchange of power between the microgrid and the main grid.

(3) When the BESS fails, the microgrid can switch smoothly to the fault operation state, which improves the reliability of the system operation.

\section{Data Availability}

The data used to support the findings of this study are included within the article.

\section{Disclosure}

This paper is an expanded version of a conference paper [28] previously published at $2^{\text {nd }}$ International Conference on 
Information Technologies and Electrical Engineering, Zhuzhou, Hunan, China, December 2019.

\section{Conflicts of Interest}

The authors declare that there are no conflicts of interest regarding the publication of this article.

\section{Acknowledgments}

This study was funded by the National Natural Science Foundation of China (51807058) and the Natural Science Foundation of Hunan Province of China (2018JJ3129).

\section{References}

[1] W.-S. Tan, M. Y. Hassan, M. S. Majid, and H. Abdul Rahman, "Optimal distributed renewable generation planning: a review of different approaches," Renewable and Sustainable Energy Reviews, vol. 18, no. 2, pp. 626-645, 2013.

[2] H. Jiayi, J. Chuanwen, and X. Rong, "A review on distributed energy resources and microgrid," Renewable and Sustainable Energy Reviews, vol. 12, no. 9, pp. 2472-2483, 2008.

[3] Q. Huang, M. L. Crow, G. T. Heydt, J. P. Zheng, and S. J. Dale, "The future renewable electric energy delivery and management (freedm) system: the energy internet," Proceedings of the IEEE, vol. 99, no. 1, pp. 133-148, 2011.

[4] Y. Liu, Y. Li, H. Liang, J. He, and H. Cui, "Energy routing control strategy for integrated microgrids including photovoltaic, battery-energy storage and electric vehicles," Energies, vol. 12, no. 2, 2019.

[5] S. Bifaretti, P. Zanchetta, A. Watson, L. Tarisciotti, and J. C. Clare, "Advanced power electronic conversion and control system for universal and flexible power management," IEEE Transactions on Smart Grid, vol. 2, no. 2, pp. 231-243, 2011.

[6] T. Esram and P. L. Chapman, "Comparison of photovoltaic array maximum power point tracking techniques," IEEE Transactions on Energy Conversion, vol. 22, no. 2, pp. 439-449, 2007.

[7] E. Koutroulis and K. Kalaitzakis, "Design of a maximum power tracking system for wind-energy-conversion applications," IEEE Transactions on Industrial Electronics, vol. 53, no. 2, pp. 486-494, 2006.

[8] S. Teleke, M. E. Baran, A. Q. Huang, S. Bhattacharya, and L. Anderson, "Control strategies for battery energy storage for wind farm dispatching," IEEE Transactions on Energy Conversion, vol. 24, no. 3, pp. 725-732, 2009.

[9] G. Delille, B. François, and G. Malarange, "Dynamic frequency control support by energy storage to reduce the impact of wind and solar generation on isolated power system's inertia," IEEE Transactions on Sustainable Energy, vol. 3, no. 4, pp. 931-939, 2012.

[10] J.-Y. Kim, J.-H. Jeon, S.-K. Kim et al., "Cooperative control strategy of energy storage system and microsources for stabilizing the microgrid during islanded operation," IEEE Transactions on Power Electronics, vol. 25, no. 12, pp. 3037-3048, 2010.

[11] H. Su, Y. Ye, Y. Qiu, Y. Cao, and M. Z. Q. Chen, "Semi-global output consensus for discrete-time switching networked systems subject to input saturation and external disturbances," IEEE Transactions on Cybernetics, vol. 49, no. 11, pp. 3934-3945, 2019.

[12] H. Su, H. Wu, and J. Lam, "Positive edge-consensus for nodal networks via output feedback," IEEE Transactions on Automatic Control, vol. 64, no. 3, pp. 1244-1249, 2019.
[13] X. Wang, G. Jiang, H. Su, and X. Wang, "Robust global coordination of networked systems with input saturation and external disturbances," IEEE Transactions on Systems, Man, and Cybernetics: Systems, pp. 1-13, 2020.

[14] T. L. Tiang, D. Ishak, and D. Ishak, "Novel MPPT control in permanent magnet synchronous generator system for battery energy storage," Applied Mechanics and Materials, vol. 110-116, pp. 5179-5183, 2011.

[15] S. Adhikari and F. Li, "Coordinated V-f and P-Q control of solar photovoltaic generators with MPPT and battery storage in microgrids," IEEE Transactions on Smart Grid, vol. 5, no. 3, pp. 1270-1281, 2014.

[16] A. Q. Huang and J. Baliga, "FREEDM system: role of power electronics and power semiconductors in developing an energy internet," in Proceedings of the 21st International Symposium on Power Semiconductor Devices \& IC's (ISPSD), pp. 9-12, IEEE, Barcelona, Spain, 2009.

[17] C. Xu, Y. Zhao, B. Qin, and H. Zhang, "Adaptive synchronization of coupled harmonic oscillators under switching topology," Journal of the Franklin Institute, vol. 356, no. 2, pp. 1067-1087, 2019.

[18] C. Xu, H. Su, and C. Liu, "Robust adaptive synchronization of complex network with bounded disturbances," Advances in Difference Equations, vol. 2019, no. 1, p. 483, 2019.

[19] Z. Lv, L. M. Wu, Z. H. Liu, L. Liu, Z. Song, and L. Sun, "Energy router with virtual inertia control schemes and concurrency operation," Concurrency and Computation: Practice and Experience, vol. 31, no. 9, p. e4715, 2018.

[20] F. Jiang, Y. Li, C. Tu, Q. Guo, and H. Li, "A review of series voltage source converter with fault current limiting function," Chinese Journal of Electrical Engineering, vol. 4, no. 1, pp. 36-44, 2018.

[21] H. Liu, Research on Control Strategy of Electronic Power transformer, Huazhong University of Science \& Technology, Wuhan, China, 2009.

[22] H. Long, J. L. Li, and K. F. He, "Research on double-closed-loop control for the frequency converters in parallel operation," Advanced Materials Research, vol. 722, pp. 503-506, 2013.

[23] D. Segaran, B. P. McGrath, D. G. Holmes et al., "Adaptive dynamic control of a bi-directional DC-DC converter," in Proceedings of the 2010 IEEE Energy Conversion Congress and Exposition, pp. 1442-1449, Atlanta, GA, USA, September 2010.

[24] J. Chen and T. O’Donnell, "Parameter constraints for virtual synchronous generator considering stability," IEEE Transactions on Power Systems, vol. 34, no. 3, pp. 2479-2481, 2019.

[25] J. Chen, M. Liu, F. Milano, and T. O’Donnell, “Placement of virtual synchronous generator controlled electric storage combined with renewable generation," in Proceedings of the 2019 IEEE Milan PowerTech, Milano, Italy, June 2019.

[26] L. Gao, S. Liu, and R. A. Dougal, "Dynamic lithium-ion battery model for system simulation," IEEE Transactions on Components \& Packaging Technologies, vol. 25, no. 3, pp. 495-505, 2002.

[27] U. Tamrakar, D. Galipeau, R. Tonkoski, and I. Tamrakar, "Improving transient stability of photovoltaic-hydro microgrids using virtual synchronous machines," in Proceedings of the IEEE Eindhoven PowerTech, pp. 1-6, Eindhoven, Netherlands, July 2015.

[28] L. Zheng, J. Wang, and J. Zeng, "Cooperative control strategy of energy router and energy storage system in microgrid," in Proceedings of the 2nd International Conference on Information Technologies and Electrical Engineering (ICITEE2019), pp. 1-7, Changsha, China, December 2019. 